\title{
Clinical efficacy of retrograde urethrography-assisted urethral catheterization after failed conventional urethral catheterization
}

\author{
Si Hyun Kim, Hee Jo Yang, Doo Sang Kim, Chang Ho Lee, Youn Soo Jeon and Ki Hong Kim*
}

\begin{abstract}
Background: Several approaches for urethral catheterization after the failure of initial urethral catheterization have been introduced. However, standard procedures regarding what should be done after failed conventional urethral catheterization have been not established. Therefore, we investigated the clinical efficacy of retrograde urethrography (RGU)-assisted urethral catheterization after failed conventional urethral catheterization.

Methods: Between July 2015 and July 2018, 136 patients who underwent RGU-assisted urethral catheterization after failed conventional urethral catheterization were included in this retrospective study. Patients' clinical data, such as age, catheterization site, and previous history of urologic operations, were collected and assessed via chart review. Univariate and multivariate logistic regression analyses were performed to identify predictive factors for the failure of this procedure.

Results: Of the 136 patients, 94 (69.1\%) experienced successful RGU-assisted urethral catheterization. Having a previous history of urologic operations, such as urethrotomy and transurethral prostatectomy, was identified as an independent predictive factor for the failure of RGU-assisted urethral catheterization (odds ratio $=9.453,95 \%$ confidence interval $=2.703-33.063, p<0.001$ ).

Conclusions: RGU-assisted urethral catheterization can be one of the modalities for providing successful catheterization after failed conventional urethral catheterization. We believe that RGU-assisted urethral catheterization can be an effective procedure if patients have no previous history of urologic operations, such as urethrotomy and transurethral prostatectomy. Trial registration Soonchunhyang university institutional review board approval (No. 2018-08-021).
\end{abstract}

Keywords: Retrograde urethrography, Urethral catheterization, Urethral injury

\section{Background}

Urethral catheterization is one of the most common urologic procedures. For various reasons, this procedure is performed on about a quarter of inpatients [1]. However, clinicians are sometimes faced with unexpected difficulties during urethral catheterization (DDUCs). Although DDUCs are not common, if this is not properly handled,

\footnotetext{
*Correspondence: urokim@schmc.ac.kr

Department of Urology, Soonchunhyang University Cheonan Hospital, Soonchunhyang University College of Medicine, 31 Suncheonhyang 6-gil, Dongnam-gu, Cheonan 31151, Korea
}

they could cause critical problems, such as urethral injury [2]. Complications associated with urethral injury are significant hazards of urinary catheterization. This causes patients to unnecessarily increase hospitalization and pay extra-medical expenses [3]. A recent study has reported that urethral catheter-related injuries occur in $1.4 \%$ of urethral catheterization patients [4], and the cost of managing these injuries was about $\$ 371,790$ [5].

Further, there are many factors that can cause failed catheterization [6]. Common causes of DDUCs for normal urethras include tight external sphincters caused by anxiety in patients and poor catheterization technique. 
Additionally, pathologic causes include urethral stricture, phimosis, edema, bladder neck contracture, prostate cancer, false passages, and benign prostatic hypertrophy, among others [7]. Among them, most urethral injuries, particularly false passages, occur during initial catheterization. This is because external sphincter contraction occurs when the catheter enters the bulbous membranous urethra. Injuries are sometimes caused by the balloon when the catheter is not fully inserted or the catheter tip itself causes incorrect passage [4]. In these cases, the retrial of catheterization with conventional blind technique could further damage the urethra [8].

Several approaches for urethral catheterization after the failure of initial urethral catheterization have been introduced [7]. However, standard procedures regarding what should be done after failed conventional urethral catheterization have been not established. Therefore, we investigated the clinical efficacy of urethral catheterization under retrograde urethrography (RGU) guidance after failed conventional urethral catheterization and attempted to identify factors for the success of this procedure.

\section{Methods}

\section{Study design}

A total of 136 consecutive male patients who underwent urethral catheterization under RGU guidance between July 2015 and July 2018 were included in this retrospective study. All patients underwent RGU-assisted urethral catheterization after failed conventional urethral catheterization. After institutional review board approval (No. 2018-08-021) was granted for this study, we conducted a retrospective chart review of the included patients. All patients received written informed consent and agreed prior to the procedure. All procedures in this study were performed in accordance with the ethical standards of the institutional and national research committee and with the 1964 Helsinki declaration.

\section{RGU-assisted urethral catheterization procedure}

RGU using a fluoroscope was performed during urethral catheterization. Urethral catheterization was performed using a 18 French sized three-way Foley catheter with a hole that was used for continuous irrigation. Prior to the procedure, the operator wore protective equipment against radiation exposure. To start the procedure, a fluoroscope was placed in front of patients. Then, a hydrophilic guide wire (Terumo Radiofocus ${ }^{\circledR}$ Guide wire Angled, $0.35^{\prime \prime}, 150 \mathrm{~cm}$ ) was inserted into bladder through the hole of the catheter under fluoroscopic guidance. Next, a 60-mL enema syringe was filled with 1:2 mixture of contrast solution and normal. Under RGU guidance, the syringe was then connected at the drainage hole of the Foley catheter and pressure was applied to the urethra. Finally, catheterization was completed through this guide wire (Fig. 1).

\section{Patients' clinical data and statistical analysis}

The following clinical data were assessed: age, past history, previous history of urologic operations, medication for lower urinary tract symptoms, site of catheterization, reason for catheterization, history of trauma, and RGU finding. Baseline characteristics were compared via ANOVA for continuous variables and via chi-square test for categorical variables. Univariate and multivariate logistic regression analyses were performed to identify predictive factors for the failure of RGU-assisted urethral catheterization. The two-sided $\mathrm{p}$-values of $<0.2$ and $<0.005$ were considered statistically significant for the univariate and multivariate analyses, respectively. All statistical analyses were performed using SPSS Statistics version 20.0.0 (IBM Corp., Armonk, NY, USA).

\section{Results}

In total, 136 patients underwent RGU-assisted urethral catheterization. Of these 136, 94 (69.1\%) experienced successful RGU-assisted urethral catheterization and 42 (30.9\%) experienced failed RGU-assisted urethral catheterization. Moreover, 18 (13.2\%) patients required suprapubic percutaneous catheterization or cystoscopyassisted urethral catheterization after failed RGU-assisted urethral catheterization, and 15 (11.0\%) were treated via urethral sound dilatation after successful RGU-assisted urethral catheterization.

Patients' baseline clinical characteristics are presented in Table 1 . In our study, 18 (13.2\%) patients had a previous history of urologic operations, including transurethral prostatectomy, radical prostatectomy, and endoscopic urethrotomy. Most events happened in the emergency room, and most of urethral catheterizations were performed for checking urine output, absolute bed rest or operation. Definite urethral injury under RGU was identified in $33(24.3 \%)$ patients.

The complete results of our univariate and multivariate analyses are presented in Table 2. These analyses identified a previous history of urologic operations as an independent predictor of failed RGU-assisted urethral catheterization (odds ratio $=9.453,95 \%$ confidence inter$\mathrm{val}=2.703-33.063, p<0.001)$.

\section{Discussion}

Since the Seldinger technique, which involves using a guide wire to obtain safe access to blood vessels, was introduced [9], it has been applied as a second-line method after failed initial urethral catheterization $[2,10$ 17]. The Seldinger technique safely to indwell urethral 

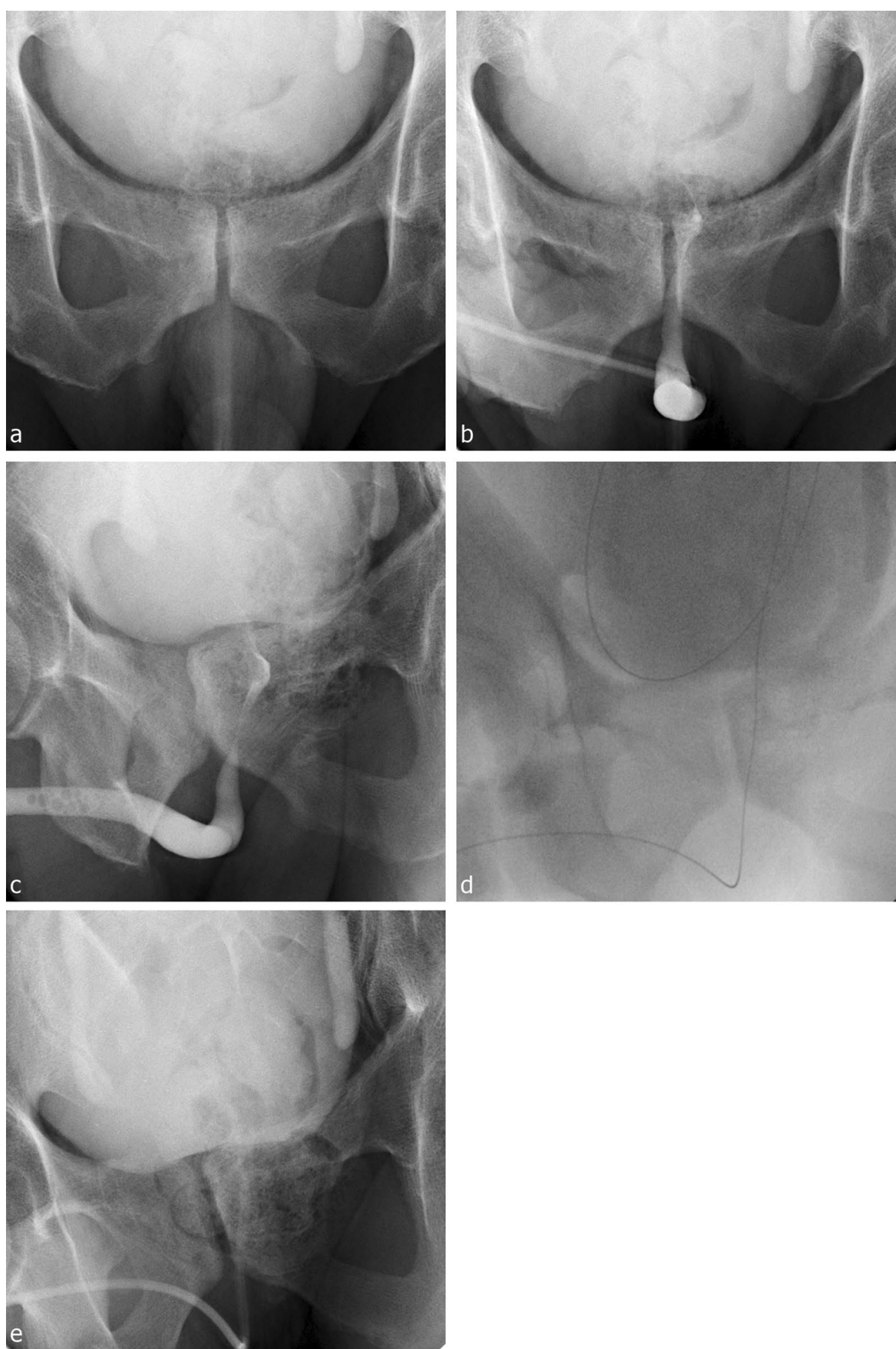

Fig. 1 Urethral catheterization under RGU. a Contrast fluid remained in the bladder after enhanced computed tomography. $\mathbf{b}$ Narrowed prostatic urethra. c An oblique image of a narrowed prostatic urethra. c A hydrophilic guide wire was inserted into bladder under fluoroscopic guidance. $\mathbf{d}$ Foley catheter was inserted into the bladder using a hydrophilic guide wire 
Table 1 Patients characteristics

\begin{tabular}{lc}
\hline Age & $\mathbf{7 6 . 0}$ \\
& $\mathbf{( 6 5 . 0 -}$ \\
& $\mathbf{8 1 . 0 )}$ \\
\hline Past history & \\
Cerebral vascular disease & 29 \\
Heart disease & 28 \\
Pulmonary disease & 47 \\
Diabetes & 20 \\
Hypertension & 50 \\
Chronic renal failure & 10 \\
Previous urologic procedure & 18 \\
Medication for LUTS & 56 \\
Site & \\
ER & 89 \\
Ward & 47 \\
Reason for catheterization & \\
AUR & 23 \\
Routine catheterization & 113 \\
Trauma & \\
Perineal & 122 \\
None & 11 \\
Others & 33 \\
RGU finding & \\
Except urethral rupture & \\
Urethral rupture & \\
\hline
\end{tabular}

catheter can be classified according to whether cystoscopy is used or not.

Urethral catheterization using only hydrophilic guide wires for DDUCs has been introduced [11-17]. The insertion of a hydrophilic guide wire, which helps to lead the urethral catheter to the correct location, can be performed without causing trauma to the urethra and with minimal pain to the patient [13]. Unfortunately, this technique is often unsuccessful making a false passage or tissue damage, because the location of guide wire could not be affirmed [11].

Recently, a novel Foley catheter that integrates a hydrophilic guide wire has also been introduced [2]. Physicians have advocated for the use of this device by reporting that it is relatively simple to use and its use results in high success rates with a $0 \%$ incidence rate of adverse events for nurse-led male urethral catheterization. However, it is difficult to guarantee a $100 \%$ success rate for the general population because this novel catheter has not been used in cases where slight resistance during urethral catheterization was present.

There have also been several reports about performing urethral catheterization via the Seldinger technique with a flexible cystoscope $[10,18]$. The authors of such a report stated that the success rate of this method was $96 \%$ [10]. Further, Villanueva et al. reported on the common approaches that are performed when initial urethral catheterization fails [19]. Based on an online survey, urologic residents most commonly resorted to flexible cystoscopy as opposed to the blind placement of guide wires or filiforms/followers after trying one or more urethral catheters. However, Villanueva et al. mentioned that urethral catheterization with flexible cystoscopy assistance is limited in terms of economics and time saving. Further, it is important to consider the learning curve for performing flexible cystoscopy. MacKenzie et al. reported that acceptable flexible cystoscopy was achieved by the 122nd procedure [20]. In other words, this procedure can only be performed with a certain level of expertise.

Other second-line urethral catheterization techniques have also been reported aside from the Seldinger technique. One such technique involves using pressure to open the urethra [21]. For this technique, a syringe filled with normal saline or sterile water is attached to behind of a typical catheter, drainage channel. By pressing the plunger of the syringe, the hydrostatic effect of the fluid helps to separate the prostatic lobes, thereby providing the tip of the catheter easier passage into the bladder [21].

Moreover, Kameda et al. attempted using transabdominal ultrasonography as a second-line urethral catheterization method [22]. They evaluated whether trans-abdominal ultrasonography could show the tip of a urethral catheter and whether trans-abdominal ultrasound-guided catheterization with transrectal pressure could result in successful catheterization for male patients in whom performing standard catheterization was difficult. However, this method is difficult to perform without an experienced physician.

Minagawa et al. reported that trans-rectal ultrasonography can be useful for DDUCs [6]. It could provide more clear findings regarding the anatomy of the male urethra. However, this procedure can only be performed by experienced physicians who understand trans-rectal ultrasound findings.

In a study including 10 patients, Athanasopoulos et al. suggested that using a ureteral access sheath for urethral dilation was helpful for the catheterization of difficult urethral strictures [23]. They mentioned that the use of both a hydrophilic guide wire and ureteral sheath proved their technique's efficacy and resulted in the atraumatic characteristics observed within the ureteral lumen. However, while successful catheterization was achieved in all patients, only a small number of patients were enrolled.

RGU-assisted urethral catheterization is a method that combined the following techniques: the Seldinger technique, fluoroscopy, and the application of hydrostatic pressure to the urethra. Therefore, the greatest advantage 
Table 2 Predictive factors for the failure of urethral catheterization under RGU

\begin{tabular}{|c|c|c|}
\hline \multicolumn{3}{|l|}{ Univariate analysis } \\
\hline Variables & OR & $p$ value \\
\hline \multicolumn{3}{|l|}{ Age } \\
\hline Continuous & $0.985(0.957-1.014)$ & 0.311 \\
\hline Categorized & $1.198(0.578-2.481)$ & 0.627 \\
\hline \multicolumn{3}{|l|}{ Past history } \\
\hline Cerebral vascular disease & $1.175(0.488-2.830)$ & 0.718 \\
\hline Heart disease & $1.024(0.416-2.518)$ & 0.959 \\
\hline Pulmonary disease & $0.603(0.271-1.340)$ & 0.214 \\
\hline Diabetes & $0.657(0.221-1.953)$ & 0.450 \\
\hline Hypertension & $1.187(0.554-2.545)$ & 0.659 \\
\hline Chronic renal failure & $0.219(0.027-1.794)$ & 0.157 \\
\hline Previous urologic procedure & $8.200(2.671-25.177)$ & $<0.001$ \\
\hline Medication for LUTS & $0.746(0.349-1.597)$ & 0.451 \\
\hline Site (ER vs GW) & $0.674(0.306-1.485)$ & 0.328 \\
\hline Reason for catheterization (AUR vs routine) & $0.514(0.205-1.289)$ & 0.156 \\
\hline Perineal trauma (vs none) & $0.472(0.098-2.287)$ & 0.351 \\
\hline RGU finding (others vs rupture) & $0.640(0.261-1.568)$ & 0.329 \\
\hline \multicolumn{3}{|l|}{ Multivariate analysis } \\
\hline Variables & OR & $p$ value \\
\hline Chronic renal failure & $0.211(0.022-2.021)$ & 0.177 \\
\hline Previous urologic procedure & $9.453(2.703-33.063)$ & $<0.001$ \\
\hline Reason for catheterization (AUR vs routine) & $0.805(0.279-3.320)$ & 0.688 \\
\hline
\end{tabular}

of this method is that it allows clinicians to clearly identify the anatomy of the urethra and evaluate the position of the guide wire. Moreover, the catheter tip could be easily inserted into the bladder because the application of hydrostatic pressure of the mixture of contrast solution and normal saline during RGU, to the urethra helped to separate the prostatic lobes. In summary, RGU-assisted urethral catheterization is a method that provides a combination of the advantages of previously reported methods. This procedure is relatively simple to implement, whereas urethral catheterization through flexible cystoscopy requires an experienced urologist.

Previous urological procedures were identified as an independent predictor for the failure of this method in multivariate analysis. Prostate transurethral resection, visual internal urethral resection, prostate holmium laser resection, and simple or radical prostatectomy are common surgeries in this category. The authors contend that these procedures resulted in an anatomical change in the urethra that the Foley catheter or guide wire could not pass through. If the patient has no history mentioned above, this method is worth considering clinically.

Our study is limited because it is retrospective in nature, it assessed only single-center data, and it did not include a large number of patients. Therefore, the results of our study must be confirmed and validated with a prospective large-scale multi-center study. Further, radiation exposure was not evaluated in our study, and a successful catheterization rate of $69.1 \%$ may not be satisfactory. However, high success rates can be expected after appropriate patient selection, as a history of previous urologic operations was identified as an independent predictor of failed RGU-assisted urethral catheterization.

\section{Conclusions}

RGU-assisted urethral catheterization can be considered one of the methods for providing successful catheterization after failed conventional urethral catheterization. In particular, if there is no previous history of urologic operations, such as urethrotomy and transurethral prostatectomy, RGU-assisted urethral catheterization can be an effective procedure for DDUCs.

\section{Abbreviations}

RGU: Retrograde urethrography; DDUC: Difficult during urethral catheterization; BPH: Benign prostatic hypertrophy; LUTS: Lower urinary tract symptoms; ER: Emergency room; AUR: Acute urinary retention.

\section{Acknowledgements}

This manuscript was prepared with the assistance of EDITAGE, an Englishlanguage scientific editing company. 


\section{Authors' contributions}

Si Hyun Kim: Conceptualization, Data curation, Methodology, Validation, Investigation, Writing-original draft. Hee Jo Yang, Doo Sang Kim, Chang Ho Lee, Youn Soo Jeon: Data curation, Validation, Investigation. Ki Hong Kim: Conceptualization, Data curation, Methodology, Validation, Investigation, Writingoriginal review \& editing. All authors read and approved the final manuscript.

\section{Funding}

This work was supported by the "Soonchunhyang University Research Fund".

\section{Availability of data and materials}

The datasets used and/or analysed during the current study available from the corresponding author on reasonable request.

\section{Ethics approval and consent to participate}

This study was performed at the Department of Urology, Soonchunhyang University Cheonan Hospital, Republic of Korea. This research involving human participants was performed in accordance with the ethical standards of the institutional and national research committee and with the 1964 Helsinki declaration. Soonchunhyang university institutional review board approved this study (No. 2018-08-021). All patients were provided written informed consent and agreed that their information would be used for this retrospective study.

\section{Consent for publication}

Not applicable.

\section{Competing interests}

The authors declare that they have no competing interests.

Received: 26 October 2020 Accepted: 14 January 2021

Published online: 04 February 2021

\section{References}

1. Jain P, Parada JP, David A, Smith LG. Overuse of the indwelling urinary tract catheter in hospitalized medical patients. Arch Intern Med. 1995;155(13):1425-9.

2. Bugeja S, Mistry K, Yim IHW, Tamimi A, Roberts N, Mundy AR. A new urethral catheterisation device (UCD) to manage difficult urethral catheterisation. World J Urol. 2019;37(4):595-600.

3. Kashefi C, Messer K, Barden R, Sexton C, Parsons JK. Incidence and prevention of iatrogenic urethral injuries. J Urol. 2008;179(6):2254-7.

4. Wagner KR, Bird ET, Coffield KS. Urinary catheterization: a paradigm shift in difficult urinary catheterization. Curr Urol Rep. 2016;17(11):82.

5. Davis NF, Quinlan MR, Bhatt NR, Browne C, MacCraith E, Manecksha R, Walsh MT, Thornhill JA, Mulvin D. Incidence, cost, complications and clinical outcomes of iatrogenic urethral catheterization injuries: a prospective multi-institutional study. J Urol. 2016;196(5):1473-7.

6. Minagawa T, Suzuki T, Domen T, Yokoyama H, Ishikawa M, Hirakata S, Nagai T, Nakazawa M, Ogawa T, Ishizuka O. Modified sonourethrography assists urethral catheterization. J Med Ultrasonics (2001). 2016;43(3):443-8.

7. Villanueva C, Hemstreet GP 3rd. Difficult male urethral catheterization: a review of different approaches. IntBraz J Urol. 2008;34(4):401-11.

8. Manalo M Jr, Lapitan MC, Buckley BS. Medical interns' knowledge and training regarding urethral catheter insertion and insertion-related urethral injury in male patients. BMC Med Educ. 2011;11:73.

9. Seldinger SI. Catheter replacement of the needle in percutaneous arteriography; a new technique. Acta Radiologica (Stockholm, Sweden: 1987). 1953;39(5):368-76.

10. Beaghler M, Grasso M 3rd, Loisides P. Inability to pass a urethral catheter: the bedside role of the flexible cystoscope. Urology. 1994;44(2):268-70.

11. Blitz BF. A simple method using hydrophilic guide wires for the difficult urethral catheterization. Urology. 1995;46(1):99-100.

12. Pachler J, Frimodt-Moller C. A comparison of prelubricated hydrophilic and non-hydrophilic polyvinyl chloride catheters for urethral catheterization. BJU Int. 1999;83(7):767-9.

13. Zammit PA, German K. The difficult urethral catheterization: use of a hydrophilic guidewire. BJU Int. 2004;93(6):883-4.

14. Levy B, Charkin A. A revised guide-wire technique for urethral catheter insertion. Ann R Coll Surg Engl. 2006;88(2):228-9.

15. Tang VC, Bott SR. A better way to insert urethral catheter with guide-wire. Ann R College Surg Engl. 2006;88(7):693.

16. Kaynar M, Akand M, Goktas S. A novel cannulation technique for difficult urethral catheterization. Associazione Ricerche Urol. 2016;88(1):60-1.

17. Yuminaga Y, Kam J, Louie-Johnsun M. Multi-centre, prospective evaluation of the Seldinger technique for difficult male urethral catheter insertions by non-urology trained doctors. BJU Int. 2017;120(Suppl 3):21-7.

18. Krikler SJ. Flexible urethroscopy: use in difficult male catheterisation. Ann R Coll Surg Engl. 1989;71(1):3.

19. Villanueva C, Hemstreet GP 3rd. The approach to the difficult urethral catheterization among urology residents in the United States. Int Braz J Urol. 2010;36(6):710-5.

20. MacKenzie KR, Aning J. Defining competency in flexible cystoscopy: a novel approach using cumulative Sum analysis. BMC Urol. 2016;16(1):31.

21. Harkin DW, Hawe M, Pyper P. A novel technique for difficult male urethral catheterization. Br J Urol. 1998;82(5):752-3.

22. Kameda T, Murata Y, Fujita M, Isaka A. Transabdominal ultrasoundguided urethral catheterization with transrectal pressure. J Emerg Med. 2014:46(2):215-9.

23. Athanasopoulos A, Liatsikos EN. The use of a ureteral access sheath for the urethral dilatation and catheterization of difficult urethral strictures. Urol Int. 2009;83(3):359-61.

\section{Publisher's Note}

Springer Nature remains neutral with regard to jurisdictional claims in published maps and institutional affiliations.
Ready to submit your research? Choose BMC and benefit from:

- fast, convenient online submission

- thorough peer review by experienced researchers in your field

- rapid publication on acceptance

- support for research data, including large and complex data types

- gold Open Access which fosters wider collaboration and increased citations

- maximum visibility for your research: over 100M website views per year

At $\mathrm{BMC}$, research is always in progress.

Learn more biomedcentral.com/submissions 